\title{
MONITORIZACIÓN DE CALIDAD DE AGUAS Y CONTROL DE PROCESOS MEDIANTE SENSORES QUÍMICOS DE FIBRA ÓPTICA
}

\author{
WATER QUALITY MONITORING AND CONTROL \\ PROCESS BY CHEMICAL FIBER OPTIC SENSORS
}

\author{
Guillermo Orellana Moraleda ${ }^{1}$
}

\begin{abstract}
RESUMEN
Nuestro equipo investigador, en colaboración con la empresa Grupo Interlab, S.A. (Madrid), lleva varios años involucrado en un ambicioso proyecto que ha llevado al mercado un instrumento optoelectrónico para análisis y control medioambiental e industrial. El sistema multicanal utiliza sensores y biosensores sobre fibra óptica basados en indicadores específicos resultantes de la ingeniería molecular y fotoquímica de complejos luminiscentes de Ru(II). De esta forma, se ha conseguido un novedoso dispositivo capaz de monitorizar simultáneamente por vía óptica, in situ y en continuo, diversos parámetros esenciales en calidad de aguas y procesos industriales (oxígeno, temperatura, $\mathrm{pH}$, amonio, $\mathrm{DBO}$, hidrocarburos, detergentes y pesticidas de tipo carbamato) mediante el simple cambio de terminal sensible situado en el extremo de la fibra óptica, ya que todos los indicadores preparados a medida se excitan por la misma luz y emiten luminiscencia en la misma región espectral. La presentación detallará el camino seguido desde el diseño y preparación de estos últimos hasta la validación de campo de los dispositivos desarrollados.
\end{abstract}

Palabras clave.- Control de procesos, Luminiscencia, Ru(II), Monitoreo, Calidad de aguas.

\begin{abstract}
RESUMEN
Nuestro equipo investigador, en colaboración con la empresa Grupo Interlab, S.A. (Madrid), lleva varios años involucrado en un ambicioso proyecto que ha llevado al mercado un instrumento optoelectrónico para análisis y control medioambiental e industrial. El sistema multicanal utiliza sensores y biosensores sobre fibra óptica basados en indicadores específicos resultantes de la ingeniería molecular y fotoquímica de complejos luminiscentes de Ru(II). De esta forma, se ha conseguido un novedoso dispositivo capaz de monitorizar simultáneamente por vía óptica, in situ y en continuo, diversos parámetros esenciales en calidad de aguas y procesos industriales (oxígeno, temperatura, $\mathrm{pH}$, amonio, $\mathrm{DBO}$, hidrocarburos, detergentes y pesticidas de tipo carbonato) mediante el simple cambio de terminal sensible situado en el extremo de la fibra óptica, ya que todos los indicadores preparados a medida se excitan por la misma luz y emiten luminiscencia en la misma región espectral. La presentación detallará el camino seguido desde el diseño y preparación de estos últimos hasta la validación de campo de los dispositivos desarrollados.
\end{abstract}

Keywords.- Process control, Luminescence, Ru (II), Monitoring, Water quality.

${ }^{1}$ Investigador del Laboratorio de Fotoquímica Aplicada, Departamento de Química Orgánica, Facultad de Química, Universidad Complutense Madrid, España. 


\section{INTRODUCCION}

El impacto de la industrialización y la contaminación medioambiental asociada a ella se ha extendido virtualmente a todas las naciones $\mathrm{y}$ comunidades.

Como consecuencia de ello, la protección del medio ambiente está recibiendo una considerable atención. Cada uno de los aspectos relacionados con dicha protección requiere, en última instancia, una monitorización de parámetros físicos o químicos ${ }^{1}$.

Cualquier programa de control medioambiental (de las aguas naturales o la atmósfera, de efluentes industriales o domésticos, del vertido de residuos, etc.) debe pasar por la determinación, preferiblemente in situ, en continuo y en tiempo real, de las especies relevantes al mismo y de su magnitud o concentración.

No resulta sorprendente, por ello, que se hayan desarrollado multitud de técnicas e instrumentos en respuesta a demandas específicas de las instituciones responsables a nivel supranacional, nacional, regional o local ${ }^{2}$.

\section{TECNOLOGIAS PARA PROTECCION DEL MEDIO AMBIENTE}

En relación a las nuevas tecnologías para la protección del medio ambiente, el parlamento europeo y el consejo de la Unión Europea subrayaron hace casi ya 10 años (Decisión $\mathrm{n}^{\circ}$ 1110/94/CE), la necesidad de que el esfuerzo en I+D tecnológico se centrara, entre otras, en las tecnologías instrumentales necesarias para la observación, vigilancia e investigación ambientales.

"Ello implica, en particular, una contribución al desarrollo de las tecnologías de observación de la tierra, [...], incluyendo aquí los sensores, las tecnologías de observación y de vigilancia de los diferentes comportamientos de la biosfera, el mayor desarrollo de las tecnologías de análisis de los diferentes medios [...].

Se incluirán también todos los instrumentos relacionados con la prevención de catástrofes naturales y con el control de la contaminación provocada por instalaciones industriales".

Estas recomendaciones quedaron reflejadas en el programa "Environment and Climate" desarrollado en el curso del IV Programa Marco de Investigación y Desarrollo Tecnológico, así como en programas posteriores (e.g. "Quality of Life and Management of Living Resources" del V Programa Marco, 1998-2002).

La sensibilidad, especificidad y versatilidad de los métodos ópticos de análisis han convertido a la espectroscopía en una técnica muy popular sobre la que basar sistemas de monitorización medioambiental ${ }^{3}$.

Desafortunadamente, las características que hacen tan atractiva la espectroscopía, también han conducido (hasta la fecha) a dispositivos costosos y métodos complejos en relación, por ejemplo, a los bien establecidos sensores electroquímicos.

Los sensores químicos de fibra óptica ( $\mathrm{u}$ "optodos", figura 1), superan dichas limitaciones mediante la fabricación de instrumentos más económicos que, al mismo tiempo, sean lo suficientemente específicos, sensibles y robustos para cumplir su misión analítica en medio ambiente, aportando sus características inherentes a la utilización de guías de luz y procesos fotoquímicos o fotofísicos apropiados (carencia de consumo de analito y daños eléctricos, versatilidad, miniaturización, densidad de información transportada, tenacidad, etc.): una luz viaja hasta el extremo sensible que contiene un indicador óptico cuya absorción, reflexión o fluorescencia se modifica por el analito de interés.

\footnotetext{
${ }^{1}$ B. Dag (ed.), "The Global Environment: Science, Technology and Management", Wiley-VCH, Weinheim (Alemania), 1997.

${ }^{2}$ Ver, p. ej., el excelente informe de WS Atkins Management Consultants, "Markets for Environmental Monitoring Instrumentation", Dept. for Trade and Industry, HMSO, Londres, 1991.

${ }^{3}$ R.N. Reeve, "Environmental Analysis”, Wiley, Chichester, UK, 1994.
} 
La instrumentación optoelectrónica que utiliza tales sensores (figura 1) consta, habitualmente, de una fuente de luz cuya radiación (generalmente monocromada por algún tipo de filtro) se lanza al interior del núcleo de un conductor óptico.

La guía de luz lleva ésta hasta el terminal sensible,-verdadero "corazón" del dispositivo-, que contiene una molécula indicadora específica (reactivo) inmovilizada firmemente en un soporte polimérico. Al producirse la interacción selectiva con el analito objetivo, alguna propiedad óptica del indicador (absorción, reflectancia, fluorescencia, polarización, índice de refracción,...) experimenta un cambio medible. Así, la radiación modificada, abandona el terminal sensible y se conduce de vuelta al detector fotónico a través de la fibra óptica. La señal eléctrica del detector se amplifica convenientemente y se procesa digitalmente.

Una calibración adecuada utilizando patrones analíticos permite llevar a cabo, posteriormente, la medida in situ y en continuo de las especies químicas de interés, con tal de que la interacción sensor-analito sea de naturaleza reversible. Si se trata de un biosensor, el transductor opto-químico se acopla en el terminal sensible con un elemento de reconocimiento de origen biológico (enzima, anticuerpo e, incluso, un microorganismo intacto) que aporta especificidad a la medida analítica.

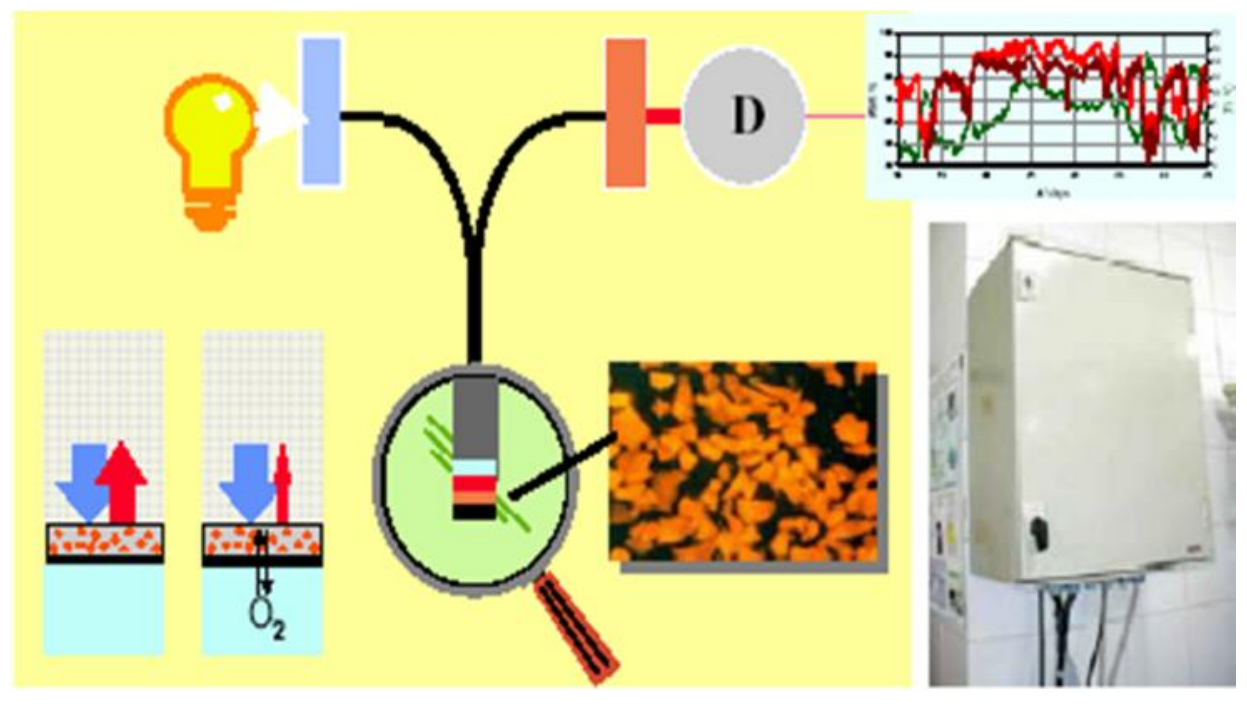

Fig. 1. Esquema simplificado de un sensor químico luminiscente sobre fibra óptica y equipo optoelectrónico multiparamétrico OPTOSEN® de Grupo Interlab (Madrid). El extremo sensible, que contienela capa reactiva con el indicador inmovilizado, es intercambiable por el usuario según el analito deseado (como ejemplo, el destinado a la medida de oxígeno disuelto en aguas).

Tradicionalmente, las medidas ópticas más utilizadas para la monitorización remota por fibra óptica ha sido la absorción de luz por las moléculas de indicador inmovilizadas (o la variación de la reflectancia difusa en el caso de indicadores soportados en materiales opacos a la radiación, pero transparentes a la entrada del analito).

No obstante, estimulada por los avances tecnológicos, la monitorización de especies químicas mediante sensores luminiscentes se va imponiendo, gracias a las ventajas decisivas que proporciona esta técnica (extrema sensibilidad, mayor selectividad, versatilidad y tiempo de vida operativa de los dispositivos resultantes).

De acuerdo con dicho principio de medida, la fuente de excitación (hoy en día diodos emisores de luz o diodos láser) envía luz de una determinada longitud de onda (o una región estrecha) al terminal sensible a través de la fibra óptica.

Las moléculas de indicador luminiscente inmovilizado absorben dicha luz y emiten radiación de mayor longitud de onda (i.e., de 
menor energía debido a las pérdidas intrínsecas derivadas de los procesos fotofísicos de excitación-relajación).

La mayoría de los indicadores que hemos desarrollado en el Laboratorio de Fotoquímica Aplicada de la UCM absorben luz violeta o azul y emiten en la región rojo-anaranjada del espectro electromagnético. De esta forma, la separación de la débil luminiscencia y la intensa excitación se ve facilitada extraordinariamente, lo que permite la utilización de filtros ópticos económicos.

En la versión más simple del dispositivo sensor, la especie química objetivo (analito) es capaz de penetrar en la membrana sensible situada en el extremo de la fibra óptica (figura 1) y colisionar con las moléculas indicadoras fotoexcitadas electrónicamente antes de que hayan tenido ocasión de volver a su estado fundamental espontáneamente (en unos pocos ns o $\mu$ s).

Como consecuencia de la colisión, tiene lugar una desactivación bimolecular del indicador competitiva con su luminiscencia, de manera que la intensidad de emisión de luz disminuye con respecto a la observada en ausencia de analito químico.

Una consecuencia obvia del proceso de desactivación molecular es la aceleración del proceso de regreso del indicador fotoexcitado, a su estado primitivo (antes de la excitación).

Por consiguiente, es posible diseñar sensores químicos sobre fibra óptica basados en la monitorización de la intensidad de luminiscencia que proviene del terminal sensible (utilizando una fuente de luz contínua para la excitación) o bien basados en la velocidad de extinción de la emisión (usando una fuente pulsada).

Las ventajas adicionales de las medidas analíticas basadas en la cinética de luminiscencia son su independencia intrínseca de (i) la cantidad de indicador inmovilizado, (ii) las fluctuaciones de la intensidad de la fuente de excitación o de la respuesta del detector y (iii) la luz ambiente (radiación continua).

La cinética de extinción de la luminiscencia de una molécula indicadora fotoexcitada se caracteriza por el denominado tiempo de vida de emisión $(\tau)$, un parámetro igual al inverso de su constante de relajación al estado fundamental.

Básicamente, hay dos métodos bien establecidos para determinar en sensores ópticos: la técnica de excitación con destello y muestreo de la señal de luminiscencia del terminal y la técnica de excitación con luz de intensidad modulada y detección, sensible a la fase, de la emisión ${ }^{4}$.

Excepto en casos muy favorables (indicadores fosforescentes), la primera es más exigente en términos de electrónica requerida que la segunda, debido a la extraordinaria rapidez del proceso de extinción de la luminiscencia $(\tau<5 \mu \mathrm{s})$.

La utilización de una unidad opto electrónica específicamente diseñada para la medida in situ, en tiempo real con sensores químicos sobre fibra óptica, capaz de interrogar diferentes terminales sensibles al mismo tiempo o en rápida secuencia y basada en la técnica de modulación de fase, debe permitir una sustancial disminución del coste asociado con la instrumentación requerida para medidas de terminales luminiscentes y un considerable aumento de la estabilidad de la medida.

En los últimos 20 años, hemos asistido prácticamente al nacimiento, infancia y llegada a la madurez de los sensores químicos de fibra óptica en el ámbito académico mundial.

Centenares de publicaciones en este campo han descrito dispositivos y principios de medida óptica de gases $(\mathrm{O} 2, \mathrm{CO} 2, \mathrm{NH} 3, \mathrm{Cl} 2, \mathrm{HCN}, \ldots)$, cationes $(\mathrm{H}+, \mathrm{Na}+, \mathrm{K}+, \ldots)$, aniones $\left(\mathrm{Cl}-, \mathrm{Br}-, \mathrm{NO}_{-}-, \ldots\right)$ y contaminantes orgánicos (hidrocarburos, organoclorados,... $)^{5}$.

\footnotetext{
${ }^{4}$ J.R. Lakowicz, "Principles of Fluorescence Spectroscopy", 2a edición, Plenum, New York, 1999.

${ }^{5}$ Dos de las más exhaustivas y recientes revisiones del tema son la monografía de O.S. Wolfbeis (ed.), "Fiber Optic Chemical Sensors and Biosensors" (2 vols.), CRC Press, Boca Raton, Fla. (USA), 1991 y el capítulo de C. Cámara, M.C. Moreno y G. Orellana, Chemical Sensing with Fiberoptic Devices, en "Biosensors with Fiber Optics”, Wise \& Wingard, eds., Humana Press, Clifton, NJ (USA), pp. 29-84, 1991.
} 


\section{SENSORES QUIMICOS}

Sin embargo, el desnivel existente entre la investigación básica y la fabricación industrial y comercialización de tales dispositivos sólo se ha superado muy recientemente.

Las razones de ello pueden resumirse en los siguientes puntos:

Desconocimiento de las necesidades del usuario final.- Frecuentemente la labor "académica" investigadora en el área de los optosensores no ha tenido en cuenta los requisitos de los usuarios potenciales de la tecnología medioambiental desarrollada (analitos y niveles de concentración, determinaciones ya resueltas satisfactoriamente por otras técnicas, estudios de costes de desarrollo y del producto final, plazos, etc.) ${ }^{6}$.

Falta de versatilidad de los sensores.- Se han descrito infinidad de principios de medida óptica (por absorción, emisión, reflexión, cambio de índice de refracción, etc.), pero cada uno es específico de un analito concreto lo cual exige cambiar la fuente de luz, sistemas de selección de longitudes de onda, disposición del terminal sensible de fibra óptica, detector, etc. en cada caso, dificultando así su puesta en el mercado ${ }^{5}$.

Coste.- La mayoría de los sensores ópticos publicados hasta la fecha se han desarrollado sobre instrumentación espectroscópica de laboratorio (sofisticados espectrómetros, láseres, monocromadores, óptica, etc.), de elevado precio y prestaciones, que no es trasladable a la situación del mercado medio ambiental enormemente competitivo y de amplia difusión, lo que determina que la instrumentación para control haya de ser económica.

No existen todavía instrumentos comerciales adaptados particularmente a la naturaleza de la medida óptica concreta.

Carencia de indicadores diseñados "a medida.- la mayoría de grupos investigadores en esta área se ha centrado fundamentalmente en los aspectos analíticos del desarrollo de sensores, utilizando indicadores ópticos comercialmente asequibles.

Sólo una colaboración multidisciplinar con químicos expertos en síntesis orgánica/inorgánica y en la fotofísica y fotoquímica de moléculas ópticamente activas hace posible diseñar principios de medida específicos para un parámetro físico o químico, con los requisitos imprescindibles de fotoestabilidad del indicador, sensibilidad al analito y anclaje al soporte polimérico que constituye el terminal sensible (ausencia de "lavado" o pérdida ${ }^{7}$ ).

Ausencia de validación analítica.- Los trabajos publicados sobre sensores químicos de fibra óptica sólo se han ensayado en condiciones de laboratorio (matrices "limpias", falta de interferentes y de las drásticas condiciones de la medida in situ, determinaciones analíticas de una única jornada de trabajo o muy pocos días, etc.).

No se han llevado a cabo ensayos de monitorización medioambiental in situ, durante un número de meses, en diferentes estaciones del año y localizaciones, y con las sondas ópticas sujetas al entorno real agresivo (e.g. "biofouling", abrasión, ensuciamiento,.. ${ }^{8}$ ).

${ }^{6}$ F. Colin y P. Quevauvillier (Eds.), "Monitoring of Water Quality: The Contribution of Advanced Technologies", Elsevier, Amsterdam, 1998.

7 (a) J.R. Lakowicz (ed.), "Topics in Fluorescence Spectroscopy", Vol. 4: "Probe Design and Chemical Sensing", Plenum, New York, 1994; (b) G. Orellana y D. García-Fresnadillo, "Environmental and industrial optosensing with tailored luminescent Ru(II) polypyridyl complexes", en "Optical Sensors: Industrial, Environmental and Diagnostic Applications" (Springer Series on Chemical Sensors and Biosensors, Vol. 1), R. Narayanaswamy y O.S. Wolfbeis, Eds., Springer Verlag, Berlin-Heidelberg, 2003.

${ }^{8}$ C. Cámara, C. Pérez-Conde, M.C. Moreno-Bondi y C. Rivas, "Fibre-Optical Sensors Applied to Field Measurements", en "Quality Assurance for Environmental Analysis: Method Evaluation within the Measurements and Testing Programme (BCR)", Ph. Quevauviller, E.A. Maier y B. Griepink (eds.), Elsevier, Amsterdam 1995; pp. 165-192. 
Una dedicación conjunta de fabricantes de instrumentación opto-electrónica, de expertos en análisis medioambiental, de organismos oficiales de validación y de los usuarios finales es, por tanto, imprescindible para "poner a trabajar" los esfuerzos conjuntos de espectroscopistas, fotoquímicos, químicos orgánicos e inorgánicos, analíticos e ingenieros en investigación básica y aplicada. El proyecto llevado a cabo en nuestro equipo de la UCM durante los últimos 7 años ha pretendido específicamente abordar dichos aspectos, gracias a la experiencia combinada de los participantes universitarios e industriales y al liderato en sus respectivas áreas de actividad.

\section{PARTE EXPERIMENTAL}

El equipo optoelectrónico desarrollado por Grupo Interlab y la UCM (OPTOSEN®), es un instrumento elaborado ex-profeso para determinaciones analíticas medioambientales in situ por fibra óptica utilizando la familia de indicadores luminiscentes ingeniados y patentados por el Laboratorio de Fotoquímica Aplicada de la UCM (ver lista de referencias). Dichos indicadores pertenecen al grupo de los compuestos de coordinación de rutenio (II) con ligandos poliazaheteroaromáticos quelatantes (figura 2$)^{9}$. Entre sus ventajas, de cara a la fabricación de terminales sensibles para medidas de luminiscencia por fibra óptica, se encuentran: una fuerte absorción en la región azul del espectro electromagnético, intensa emisión roja, largos tiempos de vida de emisión $(0,2$ a 7 microsegundos), una considerable fotoestabilidad (comparados con las moléculas fluorescentes indicadoras puramente orgánicas) y, sobre todo, la posibilidad de "sintonizar" finamente sus propiedades fotofísicas y fotoquímicas a través del molécula indicadora (ver lista de referencias). diseño molecular de los ligandos heterocíclicos, coordinados al centro metálico. Como se detallará en la presentación, la modificación química de los ligandos orgánicos, permite (i) ajustar la carga eléctrica neta global del complejo metálico indicador $(\mathrm{n}+, 0, \mathrm{o} \mathrm{m}-)$; (ii) proporcionar grupos funcionales para el anclaje del indicador al soporte polimérico; (iii) hacer efectiva o incrementar su interacción con el analito diana o evitar interferencias químicas; (iv) alterar la solubilidad del complejo; (v) variar las propiedades espectroscópicas y fotofísicas del indicador luminiscente. Incorporados en una membrana indicadora situada en el extremo terminal de un haz bifurcado de fibras ópticas, la determinación analítica puede realizarse in situ con sensores de durabilidad, versatilidad y precio competitivos o superiores a los actualmente en el mercado.

Para aumentar la sensibilidad, precisión, estabilidad, robustez y versatilidad de la medida con tales indicadores, se ha desarrollado un instrumento (OPTOSEN@, figura 1) robusto y provisto de sistemas de comunicación remota capaz de monitorizar la luminiscencia de los mismos por fibra óptica a través de la técnica de detección sensible a la fase ${ }^{10}$. La luminiscencia de un indicador excitado con luz modulada sinusoidalmente, aparece también modulada de igual forma pero desfasada respecto a la radiación incidente; la magnitud de dicho desfase es función del tiempo de vida del estado excitado (emisor) del indicador luminiscente el cual, a su vez, es función del entorno físico-químico que rodea al mismo. Es posible, realizar la cuantificación de un analito dado, con tal de que éste experimente algún tipo de interacción con la molécula indicadora (ver lista de referencias).

\footnotetext{
${ }^{9}$ Aunque pueda parecer exótico el recurrir a un metal como el $\mathrm{Ru}(\mathrm{II})$, la presencia de éste confiere las propiedades únicas al compuesto de coordinación resultante. Si se emplea Fe(II), metal mucho más abundante situado en el mismo grupo que el rutenio, el complejo resultante es fuertemente coloreado pero no luminiscente. Si se reemplaza por Os(II), metal situado inmediatamente debajo del rutenio en la Tabla Periódica de los Elementos, el complejo resultante de la coordinación a ligandos poliazaheterocíclicos es mucho menos luminiscente y presenta un tiempo de vida de emisión considerablemente inferior (ver ref. 7b).

${ }^{10}$ El sistema OPTOSEN $®$ y los correspondientes terminales sensibles luminiscentes intercambiables se fabrica y comercializa en la actualidad por Grupo Interlab, S.A. (Madrid, España) (www.interlab.es; tel. +34913589 611; fax +34-913 589 482).
} 


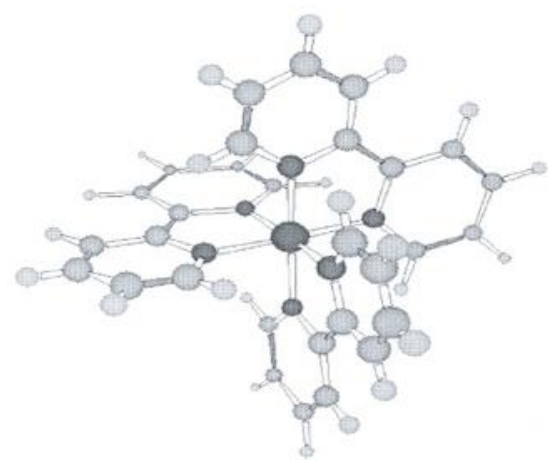

Fig. 2 Estructura tridimensional de un típico complejo luminiscente de $R u(I I)$ con ligandos poliaazaheterocíclicos quelatantes (en la figura, el ligando 2,2'-bipiridina), utilizados por nuestro equipo investigador para la fabricación de sensores y biosensores químicos sobre fibra óptica para el control de calidad de aguas. Los 8 átomos directamente unidos al centro metálico son nitrógeno; el resto constituye el esqueleto carbonado y los hidrógenos directamente unidos al mismo.

Como se ha indicado más arriba, la adecuada ingeniería molecular de los complejos luminiscentes de $\mathrm{Ru}(\mathrm{II})$ permite "sintonizar" las propiedades del indicador para una respuesta óptima a una especie química en particular ${ }^{7 \mathrm{~b}}$. Por ejemplo, la inherente sensibilidad al oxígeno molecular de tales indicadores, cuyo estado electrónico excitado de energía más baja se ve eficazmente desactivado por dicho gas, puede incrementarse notablemente introduciendo, en la estructura básica de 2,2'-bipiridina o 1,10fenantrolina, anillos bencénicos conjugados en posición 4(7) del anillo de piridina coordinado al metal (figura 2). De esta manera se prolonga notablemente el tiempo de vida del estado excitado del complejo luminiscente $\mathrm{y}$ aumentan las probabilidades de colisión con el $\mathrm{O} 2^{11}$.

La desactivación del estado excitado es rápida y completamente reversible, permitiendo con ello la medida en continuo e in situ de la concentración de $\mathrm{O} 2$ disuelto $(0,04-35 \mathrm{mg} \mathrm{L}-1)$ o en la atmósfera $(0,03-100 \%)$ con el mismo sensor (lo que no puede realizarse utilizando los actuales "electrodos de

\footnotetext{
${ }^{11}$ La disminución del tiempo de vida del estado excitado (ô) de un luminóforo en presencia de un desactivador $(\mathrm{Q})$, en fase homogénea fluida, viene dada por la denominada ecuación de SternVolmer: ô0/ô $=1+\mathrm{kqô} 0[\mathrm{Q}]$, en donde el subíndice " 0 " hace referencia a la ausencia de desactivador, $\mathrm{kq}$ es la constante de velocidad del proceso bimolecular de desactivación y $[\mathrm{Q}]$ es la
}

oxígeno"), bastando realizar el calibrado en uno u otro medio.

Por otra parte, la utilización de determinados soportes poliméricos, membranas semipermeables y/o ligandos heterocíclicos quelatantes provoca una sustancial disminución de la desactivación del complejo luminiscente por el oxígeno.

La sensibilidad al $\mathrm{pH}$ del agua se logra mediante la incorporación de ligandos poliazaheterocíclicos dotados de átomos de nitrógeno que no participan en la coordinación al centro metálico o grupos con características ácido-base en posiciones adecuadas de los anillos heterocíclicos (hidroxilo, carboxilo, amino,...).

La transferencia de ión hidrógeno a o desde el complejo metálico en estado electrónico excitado abre una vía de desactivación competitiva con la luminiscencia del indicador. Ello se manifiesta en una disminución o un aumento (según la estructura química del ligando) del tiempo de vida de emisión al aumentar el pH del medio.

concentración de desactivador en la disolución. La inmovilización del luminóforo en un soporte sólido habitualmente provoca el alejamiento de la linealidad en la ecuación de Stern-Volmer. Una explicación más detallada se encuentra en la ref. $7 b$. 
Mediante un correcto calibrado, la respuesta sigmoidal de los diversos indicadores luminiscentes diseñados y sintetizados en nuestro laboratorio permite realizar la determinación del $\mathrm{pH}$ en el intervalo deseado (que se extiende típicamente a lo largo de 4-5 unidades de $\mathrm{pH}$ ) mediante sensores ópticos (figura 3 ).

La figura 3, muestra la determinación simultánea, continua e in situ del oxígeno disuelto, el $p H$ y la temperatura con sensores de fibra óptica en un pequeño curso fluvial. El equipo OPTOSEN ${ }^{\circledR}$ y los correspondientes optodos se han instalado durante varios meses en este lugar y en otros de España para llevar a cabo su validación de campo mediante la comparación con los sensores instalados actualmente en las mismas localizaciones.

Una traza representativa de la monitorización llevada a cabo durante una semana típica muestra las variaciones diarias y ocasionales de los niveles de analito.
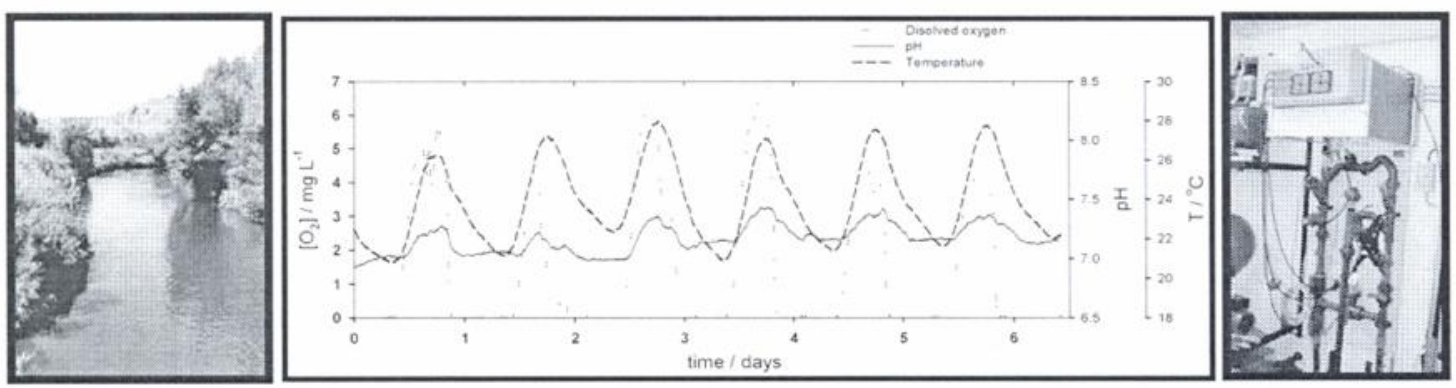

Fig. 3 Monitorización in situ, continua y simultánea de oxígeno disuelto, $p H$ y temperatura con tres de los 8 canales de fibra óptica del equipo OPTOSEN囚, en un pequeño río en los alrededores de Madrid.

Las medidas de amonio en aguas (o de bicarbonato en aguas y dióxido de carbono en fase gaseosa) pueden llevarse a cabo si se utiliza una membrana permeable exclusivamente al gas para separar la fase sensible al $\mathrm{pH}$ (un hidrogel que contiene el complejo indicador) situada en el extremo de la fibra óptica. De esta manera, se desvía una pequeñísima cantidad del agua hacia el sensor y se añade automáticamente una disolución concentrada de hidróxido sódico. La presencia de una base fuerte convierte cuantitativamente el ión amonio en gas amoniaco, que atraviesa la membrana semipermeable y penetra en la fase reactiva. Allí, la entrada de amoniaco, fuertemente básico, altera el $\mathrm{pH}$ de dicha fase, lo cual afecta dramáticamente a la luminiscencia del complejo de $\mathrm{Ru}(\mathrm{II})$. El cese de la entrada de amoniaco provoca la salida de éste del extremo sensible y el tiempo de vida de emisión del indicador recupera su valor de partida. La sensibilidad $(0,1-500 \mathrm{mg} \mathrm{L}-1)$ y tiempo de respuesta del optodo $(<120 \mathrm{~s})$ pueden seleccionarse a voluntad en función de la concentración de indicador en la fase reactiva, la concentración del electrolito ( $\mathrm{NH} 4 \mathrm{Cl}$ ) en la misma, la membrana permeable al gas y el soporte polimérico del indicador.

Nuestro equipo investigador en la UCM ha venido trabajando también, en los últimos años, en el desarrollo de un sensor luminiscente para la medida de la demanda bioquímica de oxígeno (DBO) de las aguas en continuo utilizando el monitor OPTOSEN $®$. Actualmente, el parámetro recogido en la legislación, como un indicador de la calidad de las aguas en función de la cantidad de materia orgánica presente en las mismas, es la $\mathrm{DBO}^{5}$. Dicho parámetro se determina a través de la medida, a lo largo de cinco días, del consumo de oxígeno de una muestra de agua colocada en un recipiente hermético e inoculada por una cantidad dada de biomasa. La cantidad de materia orgánica de la muestra de agua (en mg L-1) se calcula por comparación con el consumo de oxígeno que produce una mezcla patrón de glucosa y ácido glutámico (GGA). Obviamente, este parámetro puede tener un valor punitivo al infractor causante del vertido no autorizado, pero nunca paliativo al requerir 5 días para su determinación. Por ello, el desarrollo de sensores capaces de proporcionar en 
tiempo real alguna medida proporcional a la DBO5, es de candente actualidad y del máximo interés.

El funcionamiento del optodo de DBO que hemos desarrollado en colaboración con Grupo Interlab se basa en la medida del oxígeno consumido por bacterias inmovilizadas en una membrana porosa, situada en el extremo del sensor, como resultado de la respiración aeróbica de dichos microorganismos que conlleva el consumo de materia orgánica. La membrana de biorreconocimiento está unida íntimamente a otra membrana polimérica que actúa como transductor opto-químico de oxígeno. Esta última (la membrana indicadora) contiene un complejo luminiscente de $\mathrm{Ru}(\mathrm{II})$, sintetizado en nuestros laboratorios, capaz de absorber luz azul y emitir luz roja; el tiempo de vida de la luminiscencia roja del indicador es función de la concentración de oxígeno en el medio, pudiéndose medir esta última mediante el calibrado del sensor. La luz de excitación y la emitida por la membrana indicadora se conducen a través de fibra óptica hasta el dispositivo optoelectrónico de medida. Si se mantiene un nivel constante de oxígeno en la muestra (i.e. equilibrada al aire), cuya DBO se desea medir, la concentración de oxígeno que detecta en cada momento el transductor óptico acoplado a la membrana biosensora será inversamente proporcional a la cantidad de materia orgánica que contenga el agua. De esta manera, hemos conseguido la determinación de una DBO, la cual hemos demostrado que es directamente proporcional a la DBO5 en el intervalo 1-2000 mg L-1 lo que, por ejemplo, mediante el imprescindible calibrado, nos ha permitido determinar cada 30 min la DBO en el influente de una estación depuradora de aguas residuales (EDAR, figura 4). Actualmente hemos incrementado la frecuencia de medida del sistema OPTOSEN hasta los 15 min-1, lo que permite adecuar el tratamiento secundario en la EDAR a los imprevisibles picos de entrada de materia orgánica, superiores a los valores habituales.

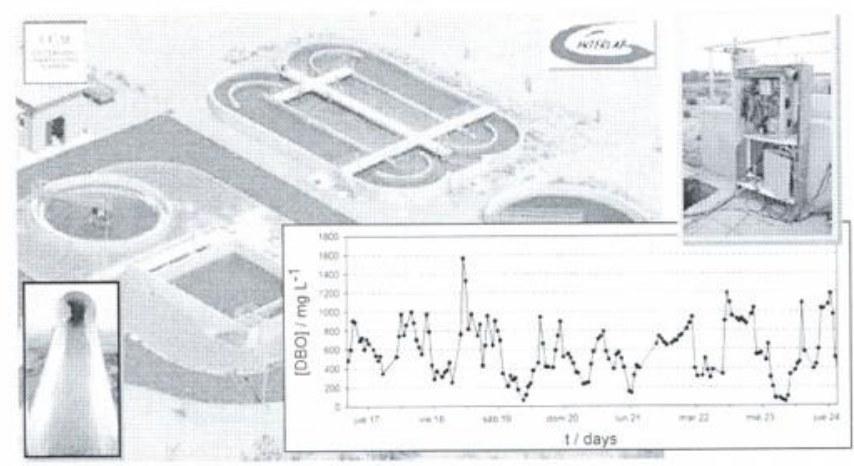

Fig. 4. Monitorización continua in situ de la demanda bioquímica de oxígeno (DBO) con el equipo OPTOSEN® y los biosensores luminiscentes desarrollados por la UCM que contienen bacterias inmovilizadas en el extremo de un transductor optoquímico de oxígeno disuelto. Cuanto mayor es la carga de materia orgánica del agua, menor es el contenido en oxígeno que detecta el transductor luminiscente.

El reconocimiento de moléculas de detergente o de hidrocarburos en las aguas tiene lugar si se introducen largas cadenas hidrocarbonadas (C14C18) en la periferia del complejo metálico y éste se ancla covalentemente sobre un soporte adecuado.

El efecto del soporte del indicador (un polímero orgánico o inorgánico) no puede despreciarse: frecuentemente el mejor complejo luminiscente indicador, como se ha deducido de investigaciones previas sobre su interacción con el analito en disolución, no proporciona un optodo útil debido a los importantes cambios de sensibilidad, al lavado del mismo o a interferencias químicas que se producen tras la inmovilización.

La determinación de pesticidas de tipo carbamato ha podido realizarse mediante un biosensor enzimático que aprovecha la inhibición de la 
actividad catalítica de la acetilcolinesterasa por estos analitos. Para ello, hemos diseñado y sintetizados complejos de $\mathrm{Ru}(\mathrm{II})$ acetilados en la parte externa de uno de sus ligandos poliazaheterocíclicos, cuya luminiscencia disminuye drásticamente al producirse la hidrólisis catalizada por la mencionada enzima. Cuanto mayor es la concentración del carbamato, más lenta es la hidrólisis del indicador. En este caso no se trata de indicadores reversibles, sino que se destruyen como resultado de la medida (cinética). Un sistema de análisis por inyección en flujo (FIA) permite realizar de manera automática las determinaciones analíticas.

Finalmente, se puede conseguir una apreciable sensibilidad a la temperatura del entorno en el que se encuentra introducido el sensor, mediante la preparación de complejos luminiscentes de $\mathrm{Ru}$ (II) con ligandos fuertemente atractores de electrones. La sensibilidad cruzada al oxígeno y a otras especies químicas mencionadas más arriba se evita mediante el atrapamiento del indicador en un polímero transparente a la luz pero impermeable a las mismas.

\section{CONCLUSIONES}

- Detección prematura de casos de contaminación

- Monitorización en general de la calidad del agua, estaciones depuradoras de aguas residuales, embalses, etc.

- Posibilidad de monitorización en tiempo real de descargas autorizadas

- Medida de descargas intermitentes procedentes de colectores de tormentas e inundaciones

- Disminución, en fin, de las necesidades de muestreo personal contínuo y análisis de laboratorio

Es evidente que las potenciales aplicaciones del dispositivo opto-electrónico y los sensores luminiscentes desarrollados no se limitan al ámbito medioambiental, sino que pueden fácilmente extenderse a la garantía de calidad de alimentos, control y análisis de procesos industriales, biotecnología, Química clínica, entre otras áreas. Algunas de ellas ya se están realizando con éxito en colaboración con los usuarios finales de la tecnología.

\section{AGRADECIMIENTOS}

El autor desea expresar su más sincero agradecimiento a la labor del equipo liderado por la Profesora María Cruz Moreno Bondi, del Dpto. de Química Analítica de la UCM, en el desarrollo de los sensores químicos sobre fibra óptica que describe este trabajo, sin cuya contribución no se hubieran alcanzado los resultados analíticos obtenidos, así como al trabajo de los muchos y excelentes investigadores pre- y post-doctorales que han realizado su trabajo en el Laboratorio de Fotoquímica Aplicada, que resulta imposible enumerar aquí. Asimismo, nuestro trabajo no hubiera sido posible sin la financiación del Ministerio de Ciencia y Tecnología español (antes Ministerio de Educación y Ciencia) (FIT-0500002001-32 y BQU2002-04515-CO2-02), la Universidad Complutense, la Comunidad Autónoma de Madrid (CAM 07M-0030-2002), la Unión Europea (BRPR-CT97-0485) y empresas privadas como Grupo Interlab, Repsol-YPF, Agilent Technologies, CESA y Physical Optics Corp., entre otras.

\section{REFERENCIAS}

(Sensores ópticos)

1. C. Cámara, M. C. Moreno, G. Orellana, Chemical Sensing with Fiberoptic Devices, en "Biosensors with Fiberoptics", D. L. Wise, L. B.Wingard, Jr. (editores), The Humana Press, Clifton, New Jersey, 1991; pp. 29-84.

2. M. C. Moreno, A. Martínez, P. Millán, C. Cámara, "Study of a $\mathrm{pH}$ sensitive optical fibre sensor based on the use of cresol red", J. Molec. Struct. 1986, 143, 553-556.

3. A. Martínez, M. Cruz Moreno, C. Cámara, "Sulfide Determination by $N, N$-Dimethyl$p$-phenylenediamine Immobilization in Cationic Exchange Resin Using an Optical Fiber System", Anal. Chem. 1986, 58, 1877-1881.

4. M. Faraldos, M. C. Moreno, C. Cámara, "Reversible optical fibre sensor to determine iron", Analusis 1988, 16, 87-91.

5. M. Cruz Moreno, M. Jiménez, C. Pérez Conde, C. Cámara, "Analytical performance of an optical $\mathrm{pH}$ sensor for acid-base titration", Anal. Chim. Acta 1990, 230, 35-40. 
6. M. C. Moreno-Bondi, O. S. Wolfbeis, M. J. P. Leiner, B. P. H. Schaffar, "Oxygen Optrode for Use in a Fiber-optic Glucose Biosensor", Anal.Chem. 1990, 62, 2377 2380.

7. M. C. Moreno-Bondi, G. Orellana, C. Cámara, O. S. Wolfbeis, "New luminescent metal complex for $\mathrm{pH}$ transduction in optical fiber sensing. Application to a CO2-sensitive device", en Chemical, Biochemical, and Environmental Fiber Sensors II, Proc. SPIE, vol 1368, 157-164, (1990).

8. G. Orellana, M. C. Moreno Bondi, "Sensor óptico" ("Optical Sensor"), Patente Española 9002740 (1990).

9. G. Orellana, M. C. Moreno-Bondi, E. Segovia, M. D. Marazuela, "Fiber-Optic Sensing of Carbon Dioxide Based on Excited-State Proton Transfer to a Luminescent Ruthenium(II) Complex", Anal. Chem. 1992, 64, 2210-2215.

10. G. Orellana, M. C. Moreno, C. de Dios, A. A. García, A. M. Gómez, "Sensor óptico luminiscente" ("Lumnescent Optical Sensor”), Patente Española 9300938 (1993).

11. J. M. Barrero, M. C. Moreno-Bondi, M. C. Pérez-Conde, C. Cámara, "A biosensor for ferric ion", Talanta 1993, 40, 1619-1623.

12. G. Orellana, M. L. Quiroga, C. de Dios, "Spectroscopy, Electrochemistry, and Photochemistry of Ruthenium(II) Complexes Containing Five- Membered Heterocyclic Chelating Ligands", Trends Inorg. Chem. 1993, 3, 109-130.

13. C. Cámara, C. Pérez-Conde, M. C. Moreno-Bondi, C. Rivas, "Fibre-Optical Sensor Applied to Field Measurements", en "Quality Assurance for Environmental Analysis: Method Evaluation within the Measurements and Testing Programme $(B C R)$ ", Quevauviller, Ph., Maier, EA., Griepink, B. (editores.), Elsevier 1995; pp; 165-192.

14. M. D. Marazuela, M. C. Moreno-Bondi, G. Orellana, "Enhanced performance of a fiber-optic luminescent $\mathrm{CO} 2$ sensor using carbonic anhydrase", Sensors \& Actuators, B: Chem. 1995, 29, 126-131.

15. G. Orellana, A. M. Gómez-Carneros, C. de Dios, A. A. García-Martínez, M. C. Moreno-Bondi, "Reversible Fiber-Optic
Fluorosensing of Lower Alcohols", Anal. Chem. 1995, 34, 2231-2238.

16. G. Orellana, C. de Dios, M. C. MorenoBondi, M. D. Marazuela, "Intensity- and Lifetime-Based Luminescence Optosensing of Carbon Dioxide", en Chemical, Biochemical and Environmental Fiber Sensors VII, A. M. Scheggi, (editora), Proc. SPIE, vol 2508, , 18-29 (1995).

17. F. J. Mingoarranz, M. C. Moreno-Bondi, D. García-Fresnadillo, C. de Dios, G. Orellana, "Oxygen-Sensitive Layers for Optical Fiber Devices", Mikrochim. Acta 1995, 121, 107-118.

18. N. I. Croitoru, M. Miyagi, G. Orellana, A. M. Scheggi, H. J. M. Sterenborg (editores) en "Medical and Fiber Optic Sensors and Delivery Systems", Proc. SPIE, vol 2631, Bellingham, WA, USA, (1996).

19. G. Orellana, D. García Fresnadillo, M. C. Moreno Bondi,, M. P. Xavier, "Método de unión a polímeros de un compuesto indicador sensible al analito, para fabricación de sensores ópticos, su dispositivo" ("Method of binding to polymers of an analyte-sensitive indicator compound in order to fabricate optical sensors, and its device") Patente Española 9602181 (1996).

20. G. Orellana, M. C. Moreno Bondi, D. García Fresnadillo, M. D. Marazuela, C. Knapp, A. M. Castro, "Sensor óptico para detergentes" ("Optical sensor for detergents"), Patente Española 9602182 (1996).

21. M. D. Marazuela, B. Cuesta, M. C. Moreno-Bondi and A. Quejido, "Free cholesterol fiber-optic biosensor for serum samples with simplex optimization", Biosensors \& Bioelectron., 1997, 12, 233240.

22. B. Schlageter, S. Pörting, J. Strassburger, M. C. Moreno-Bondi, S. E. Braslawsky, E. Oliveros, A.M. Braun, "Development of an optoacoustic sensor module for $\mathrm{pH}$ and/or $\mathrm{CO} 2$ determination in aqueous solutions"', Sensors \& Actuators, B: Chem., 1997, 38-39, 443-447.

23. G. Orellana, M. C. Moreno-Bondi, J. Delgado, M. Bedoya, A. Yáñez, J.M. Sicilia, "Sensores químicos de fibra óptica 
para análisis medioambiental", Química Hoy, 1997 (Sept.), 31-35.

24. G. Orellana, D. García-Fresnadillo, M.D. Marazuela, M.C. Moreno-Bondi, J. Delgado, J.M. Sicilia, "Fibre-Optic Chemical Sensors: From Molecular Engineering to Environmental Analytical Chemistry in the Field", en "Standards, Measurements and Testing for the Monitoring of Water Quality: The contribution of Advanced Technologies", Elsevier, 1998, 103-115.

25. M. D. Marazuela, M. C. Moreno-Bondi, G. Orellana, "Luminescence lifetime quenching of a ruthenium(II) polypyridyl dye for optical sensing of carbon dioxide", Appl. Spectrosc., 1998, 52, 1314-1320.

26. M. P. Xavier, D. García-Fresnadillo, M. C. Moreno-Bondi, G. Orellana, "Oxygen sensing in nonaqueous media using porous glass with covalently bound luminescent Ru(II) complexes", Anal. Chem., 1998, 70, 5184-5189.

27. D. García-Fresnadillo, M.D. Marazuela, M.C. Moreno-Bondi, G. Orellana, "Luminescent Nafion membranes dyed with Ruthenium(II) complexes as sensing materials for dissolved oxygen", Langmuir 1999, 15, 6451-6459.

28. M.P. Xavier, G. Orellana, M.C. MorenoBondi, J. Díaz-Puente, "Carbon dioxide monitoring in compost processes using fibre-optic sensors based on a luminescent ruthenium(II) indicator", Quim. Anal. 2000, 19, 118-126.

29. F. Navarro-Villoslada, G. Orellana, M. C. Moreno-Bondi, T. Vick, M. Driver, G. Hildebrand, K. Liefeith, "Fiberoptic Luminescent Sensors with Composite Oxygen-Sensitive Layers and AntiBiofouling Coatings", Anal. Chem. 2001, 73, 5150-5156.

30. D. García-Fresnadillo, G. Orellana, "Interaction of Sulfonated Ruthenium(II) Polypyridyl Complexes with Surfactants Probed by Luminescence Spectroscopy", Helv. Chim. Acta 2001, 84, 2708-2730.

31. M. Bedoya, M.C. Moreno-Bondi, G. Orellana, "Fluorescent Optosensor for Humidity Measurements in Air", Helv. Chim. Acta 2001, 84, 2628-2639.

32. L. M. Fraga Trillo, V. Ruiz-Santa Quiteria, G. Orellana Moraleda, A. Castro Franco,
"Sensors for the Determination of Organometallic Compounds", Eur. Patent Appl. 01500101.9 (17 Abril 2001), US Patent Appl. 10/125,006. Países a los que se ha extendido: Mexico, India, Sudáfrica, Brasil, Taiwan, Rusia.

33. M. C. Moreno-Bondi, G. Orellana, M. Bedoya, "Fibre-optic sensors for humidity monitoring", en "Optical Sensors: Industrial, Environmental and Diagnostic Applications" (Springer Series on Chemical Sensors and Biosensors, Vol. 1), R. Narayanaswamy y O.S. Wolfbeis, Eds. Springer Verlag, Berlin-Heidelberg, 2003.

\section{(Indicadores ópticos)}

34. G. Orellana, M. L. Quiroga, A. M. Braun, "Spectroscopic, electrochemical, and kinetic characterization of new ruthenium(II) tris-chelates containing fivemembered heterocyclic moieties", Helv. Chim. Acta, 1987, 70, 2073-2073.

35. G. Orellana, C. Alvarez-Ibarra, J. Santoro, "H-1 and C-13 NMR Coordinationinduced shifts in a series of tris(ádiimine)ruthenium(II) complexes containing pyridine, pyrazine, and thiazole moieties", Inorg. Chem., 1988, 27, 1025 1030.

36. G. Orellana, C. Alvarez-Ibarra, M. L. Quiroga, "New binuclear heterocyclic ligands sharing an á-diimine moiety and their ruthenium(II) trischelates", Bull. Soc. Chim. Belg., 1988, 97, 731-741.

37. G. Orellana, A. M. Braun, "Quantum,ields of 3MLCT excited state formation and triplet-triplet absorption spectra of $\mathrm{Ru}$ (II) tris-chelate complexes containing fiveand six-membered heterocyclic moieties", J. Photochem. Photobiol., A: Photochem., 1989, 48, 277-289.

38. M. C. Moreno-Bondi, G. Orellana, N. J. Turro, D. A. Tomalia, "Photoinduced electron transfer reactions to probe the structure of starburst dendrimers", Macromolecules, 1990, 23, 910-912.

39. G. Orellana, A. Kirsch-De Mesmaeker, N. J. Turro, "Ru-99 NMR of ruthenium(II) polypyridyl complexes", Inorg. Chem.,1990, 29, 882-885.

40. A. Kirsch-De Mesmaeker, G. Orellana, J. K. Barton, N. J. Turro, "Ligand-dependent 
interaction of ruthenium(II) polypyridyl complexes with DNA probed by emission spectroscopy", Photochem. Photobiol., 1990, 52, 461-472.

41. G. Orellana, A. Kirsch-De Mesmaeker, J. K. Barton, N. J. Turro, "Photoinduced electron transfer quenching of excited $\mathrm{Ru}$ (II) polypyridyls bound to DNA: The role of the nucleic acid double helix", Photochem. Photobiol. 1991, 54, 499-509.

42. G. Orellana, M. L. Quiroga, C. de Dios, "Spectroscopy, electrochemistry, and photochemistry of ruthenium(II) complexes containing fivemembered heterocyclic chelating ligands", Trends in Inorganic Chemistry, 1993, III, 109-130.

43. J.-P. Lecomte, A. Kirsch-De Mesmaeker, G. Orellana, "Photophysics of polyazaaromatic ruthenium (II) complexes interacting with DNA", J. Phys. Chem., 1994, 98, 5382-5388.

44. G. Colmenarejo, M. C. Gutiérrez-Alonso, M. Bárcena, J. M. Kelly, F. Montero, G. Orellana, "Interaction with DNA of photoactive viologens based on the 6-(2Pyridinium)-phenanthridinium structure", J. Biomol. Struc. Dyn., 1995, 12, 827-846.

45. C. Knapp, J.-P. Lecomte, A. Kirsch-De Mesmaeker, G. Orellana, "Photoinduced electron transfer from nucleotides to 6-(2Pyridinium) phenanthridinium viologens: A study by laser flash photolysis and spectroelectrochemistry", J. Photochem. Photobiol.; B: Biol., 1996, 36, 67-76.
46. D. García-Fresnadillo, Y. Georgiadou, G. Orellana, A. M. Braun, E. Oliveros, "Singlet oxygen (1.g) production by ruthenium(II) complexes containing polyazaheterocyclic ligands in methanol and in water", Helv. Chim. Acta, 1996, 79, 1222-1238.

47. G. Orellana, M. E. Jiménez, M. T. Portolés, F. Montero, M. Catalá, "Utilización de los compuestos de coordinación de rutenio que contienen dipiridofenazina como marcadores luminiscentes de la viabilidad celular" ("Use of $\mathrm{Ru}(\mathrm{II})$ coordination compounds containing dipyridophenazine as luminescent markers of cell viability"), Patente Española 9801936 (1998).

48. M.E. Jiménez-Hernández, G. Orellana, F. Montero y M.T. Portolés, "A ruthenium probe for cell viability measurement using flow cytometry, confocal microscopy, and time-resolved luminescence", Photochem. Photobiol. 2000, 72, 28-34.

49. G. Orellana y D. García-Fresnadillo, "Environmental and industrial optosensing with tailored luminescent $\mathrm{Ru}(\mathrm{II})$ polypyridyl complexes", en "Optical Sensors: Industrial, Environmental and Diagnostic Applications" (Springer Series on Chemical Sensors and Biosensors, Vol. 1), R. Narayanaswamy y O.S. Wolfbeis, Eds., Springer Verlag, Berlin-Heidelberg, 2003.

orellana@quim.ucm.es 